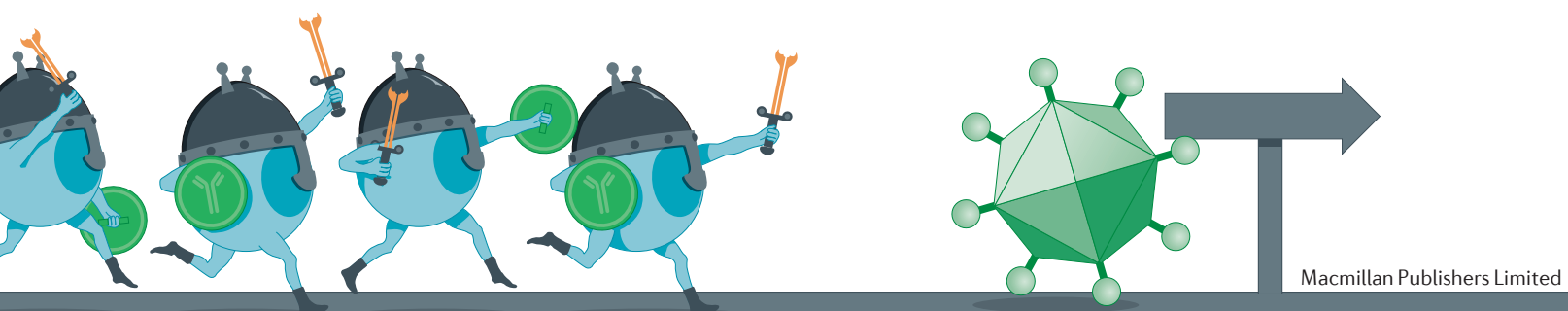

IMMUNOTHERAPY

\title{
Oncolytic viruses prime antitumour immunity
}

Oncolytic virotherapies activate an immune response and, thus, their addition has been shown to improve the outcomes of immune-checkpoint blockade (ICB) in patients with melanoma. The results of two window-of-opportunity (WOO) trials, now published in Science Translational Medicine, indicate that patients with primary brain tumours or metastases, as well as those with triple-negative breast cancer (TNBC), can also benefit from such combination therapies.

The blood-brain barrier might limit the delivery of viral particles to brain tumours. To overcome this hurdle, Adel Samson and colleagues designed a phase lb WOO surgery trial in which six adult patients with high-grade gliomas and three with brain metastases received a single intravenous dose of reovirus 3-17 days before surgical tumour resection. The median overall survival duration after reovirus infusion, 469 days (118-1,079 days), was in the expected range for patients with these malignancies. All patients had adverse events (mostly lymphopenia and flu-like symptoms), which were grade 3/4 in six patients.

Increased levels of IFN $\alpha$ and other cytokines, indicative of a systemic immune response, were detected in blood samples taken 2 days after infection (relative to baseline samples). "Delivery of reovirus to brain tumours was observed in all tested patients, resulting in increased immune cell infiltration and upregulation of PD-L1, which could prime tumours for subsequent ICB," explains Samson. On the basis of these observations, immunocompetent mice with orthotopic glioma received systemic treatment with reovirus, an ICB agent, or both. In mice that received this combination, longer survival durations were observed, together with high levels of immune-cell infiltration into tumours.

In a separate study, Marie-Claude Bourgeois-Daigneault and colleagues addressed the suboptimal outcomes of the trials of ICB that have been conducted so far in patients with TNBC. The investigators used the Maraba rhabdovirus in combination with ICB in a WOO study in mice with TNBC. "When administered before surgical resection of the primary mass, Maraba virus could efficiently control metastases and prevent relapse. Virus treatment recruited immune cells to the tumour bed and primed antitumour immunity," highlights Bourgeois-Daigneault. A complete response was not observed before tumours were surgically resected. Mice were then subjected to secondary tumour seeding, followed by ICB in some of them. Mice that had received Maraba had a better response to ICB than mice treated with ICB only.

Promising outcomes have already been observed in WOO trials in patients with breast cancer, and now Samson and colleagues have proved their feasibility in patients with brain tumours or metastases. The results discussed herein indicate that combination therapies of oncolytic viruses and ICB warrant further investigation in early clinical trials in patients with these malignancies.

Diana Romero 